\title{
The impact of vegetation on the characteristics of the flow in an inclined open channel using the piv method
}

\author{
Keramaris Evangelos \\ Department of Civil Engineering, Division of Hydraulic and Environmental Engineering, University of Thessaly, Pedion Areos, Volos, \\ Greece
}

\section{Email address:}

ekeramaris@uth.gr (K. Evangelos)

\section{To cite this article:}

Keramaris Evangelos. The Impact of Vegetation on ohe Characteristics of the Flow in an Inclined Open Channel Using the Piv Method. Water Resources and Ocean Science. Vol. 1, No. 1, 2012, pp. 1-6. doi: 10.11648/j.wors.20120101.11

\begin{abstract}
In this study, the impact of vegetation on the characteristics of the flow in an inclined open channel is studied experimentally using a Particle Image Velocimetry (PIV). This optical method of fluid visualization is used to obtain instantaneous velocity measurements related properties in the fluids. For the simulation of vegetated bed, flexible vegetation with grass with different height $(2.5 \mathrm{~cm}$ and $5 \mathrm{~cm})$ with the same porosity $(\varepsilon=0.75)$ were used. These conditions can be commonly found in systems with sediment transport. Twenty-four (24) experiments were conducted, twelve (12) with the flexible vegetation of $2.5 \mathrm{~cm}$ and twelve (12) with the flexible vegetation of $5 \mathrm{~cm}$. The slope of the channel was kept equal to $\mathrm{S}=2 \%, \mathrm{~S}=4 \%$ and $\mathrm{S}=6 \%$. The experiments were conducted in the laboratory of Hydraulics in the department of Civil Infrastructure Engineering of Alexander Technological Educational Institute of Thessaloniki, Greece. The channel has a length of $6.5 \mathrm{~m}$, width of $7.5 \mathrm{~cm}$ and height of $25 \mathrm{~cm}$. The impact of the channel slope, the vegetation height, the vegetation length and the relative vegetation depth on the characteristics of the flow is studied. Measurements of velocity were taken for horizontal channel slope at different heights using the PIV. Results show that the bed type can significantly influence the characteristics of the flow.
\end{abstract}

Keywords: Vegetation, Turbulent Flow, Experimental Analysis, Particle Image Velocimetry

\section{Introduction}

The study of the impact of vegetation on turbulent flow in an open channel has particular importance. The presence of vegetation in rivers and open channels influences significantly the velocity and the depth flow. The variations observed at the beginning of vegetation, through the length, in the end and a little after. There are many kinds of submerged vegetation which vary depending on the density, the flexibility and the height, where each of these characteristics influences the flow with different way.

Initially in reference [1] Beavers and Joseph (1967) used a porous medium with high permeability and laminar flow in a closed channel and found an empirical relationship for the interfacial slip velocity. They noticed an increase of mass flow over a permeable bed in comparison with the fluid flow over an impermeable bed.

In reference [7] Poulikakos and Kazmierczak (1987) have used laminar flow and Navier-Stokes equations for the flow and Darcy-Brinkman equations for the flow within the porous medium. They calculated analytically the inter- facial velocity and the velocity distribution above and inside the porous region.

In reference [9] Stephan and Gutknecht (2002) investigated the resistance of submerged flexible aquatic vegetation on the flow. They described the flow resistance of the natural macrophytes used by means of equivalent sand roughness and they found out that the latter as well as the zero plane displacement of the logarithmic velocity profile were of the same order of magnitude as the mean deflected plant height and increased with increasing plant height.

In reference [11] Wilson et al. (2003) carried out laboratory experiments to study the effect of flexible vegetation on open channel flow. They used two different forms of vegetation a) flexible rods of constant height and b) the same rods with a front foliage attached. The turbulence intensities indicated that the additional superficial area of the fronts alters the momentum transfer between the within-canopy and surface flow regions. Finally, this research indicated that the plant form can have a significant effect on the mean flow field and, therefore, potentially influence riverine and wetland system management strategies.

In reference [8], in the work of Prinos et al. (2003) expe- 
rimental and computational results were presented for turbulent flow above and within a porous medium. The latter was simulated with a bundle of cylindrical rods. Experimental results from the above work were used in reference [4] by Chan et al. (2007) for testing a numerical model, based on macroscopic flow characteristics, and a good agreement was found between experimental and computed results.

In reference [5] Jarvela (2005) investigated the flow structure above flexible vegetation (i.e. wheat). Velocity and turbulent characteristics were measured with a 3D Acoustic Doppler Velocimeter. Results showed that the flow above wheat crop follows a logarithmic law. The maximum values of the turbulence intensity and shear stress were found approximately at the level of the maximum observed deflected plant height. In reference [3] Carollo et al. (2005) conducted experiments in a straight flume having a bed covered with grass like vegetation in order to analyse flow resistance for flexible submerged elements. The authors concluded that a flow resistance equation, linking the friction factor with the shear Reynolds number, the depth-vegetation height ratio and the inflection degree can be established.

In reference [2] Bigillon et al. (2006) investigated the turbulent characteristics of open-channel flow under transitionally-rough wall conditions. Vertical velocity profiles, turbulent intensities and vertical fluxes of turbulent kinetic energy were investigated using a PIV over a transitionallyrough fixed bed. Results were further compared to those obtained for smooth, rough and transitionally-rough walls. The authors conclude that the PIV technique can be applied successfully to investigate turbulent open channel flow over rough bed. However the impact of vegetation in an inclined open channel is not studied.

In reference [10] Roussinova et al. (2010) carried out PIV measurements of the instantaneous velocity fields in a smooth open channel flow (Reynolds number was 21000 and the maximum velocity was $0.19 \mathrm{~m} / \mathrm{s}$ ).

More recently in reference [6] Pechlivanidis et al. (2012) investigated experimentally the turbulent characteristics of open-channel flow using Particle Image Velocimetry. Results show that velocity over the vegetation region is a function of the vegetation height and the total flow depth; velocity decreases as the vegetation height increases. In addition, we show that velocities above the vegetation region are much lower than velocities above an impermeable bed. This is due to the turbulent shear stresses and the existence of turbulence in the vegetation region, which reduce the mean velocity above the vegetation region.

In this study, the impact of vegetation on the characteristics of the flow in an inclined open channel is studied experimentally using a Particle Image Velocimetry (PIV). These conditions can be commonly found in systems with sediment transport. Twenty-four (24) experiments were conducted, twelve (12) with the flexible vegetation of $2.5 \mathrm{~cm}$ and twelve (12) with the flexible vegetation of $5 \mathrm{~cm}$. The impact of the channel slope, the vegetation height, the ve- getation length and the relative vegetation depth on the characteristics of the flow is studied. Results show that the impact of the above parameters is important on the flow characteristics before, above and after the vegetation.

\section{Experimental Procedure- Measurements}

In total twenty-four (24) experiments were conducted, twelve (12) with the flexible vegetation of $2.5 \mathrm{~cm}$ and twelve (12) with the flexible vegetation of $5 \mathrm{~cm}$. The experiments were conducted in the laboratory of Hydraulics in the department of Civil Infrastructure Engineering of Alexander Technological Educational Institute of Thessaloniki, Greece. The channel has a length of $6.5 \mathrm{~m}$, width of $7.5 \mathrm{~cm}$ and height of $25 \mathrm{~cm}$. For the simulation of vegetated bed, flexible vegetation with grass with different height $(2 \mathrm{~cm}$ and $5 \mathrm{~cm})$ with the same porosity $(\varepsilon=0.75)$ were used. The vegetations of $2.5 \mathrm{~cm}$ and $5 \mathrm{~cm}$ thickness were attached to a $1.0 \mathrm{~cm}$ thick wood, so that it remains fixed to the bed during the experiments.

Hydraulic characteristics were measured at two different total flow depths $\mathrm{h}=10$ and $15 \mathrm{~cm}$. The flow depth over the permeable bed $h^{\prime}$ was $h^{\prime}=7.5$ and $12.5 \mathrm{~cm}$ for the vegetation of $2.5 \mathrm{~cm}$ height and $\mathrm{h}^{\prime}=5$ and $10 \mathrm{~cm}$ for the vegetation of $5 \mathrm{~cm}$ height. With these conditions we kept the total flow depth constant equal to $\mathrm{h}=10$ and $15 \mathrm{~cm}\left(\mathrm{~h}=\mathrm{h}^{\prime}+\mathrm{hv}\right.$, hv : height of vegetation). The slope of the channel was kept equal to $\mathrm{S}=2 \%$, $S=4 \%$ and $\mathrm{S}=6 \%$ and the vegetation length was 150 and $300 \mathrm{~cm}$. The blades were made from plastic. The geometrical characteristics of the flow are presented in Figure 1. The experimental apparatus is presented in Figure 2. The morphology of the vegetation is illustrated in Photograph 1, and the characteristics of the experiments are presented in Table 1.

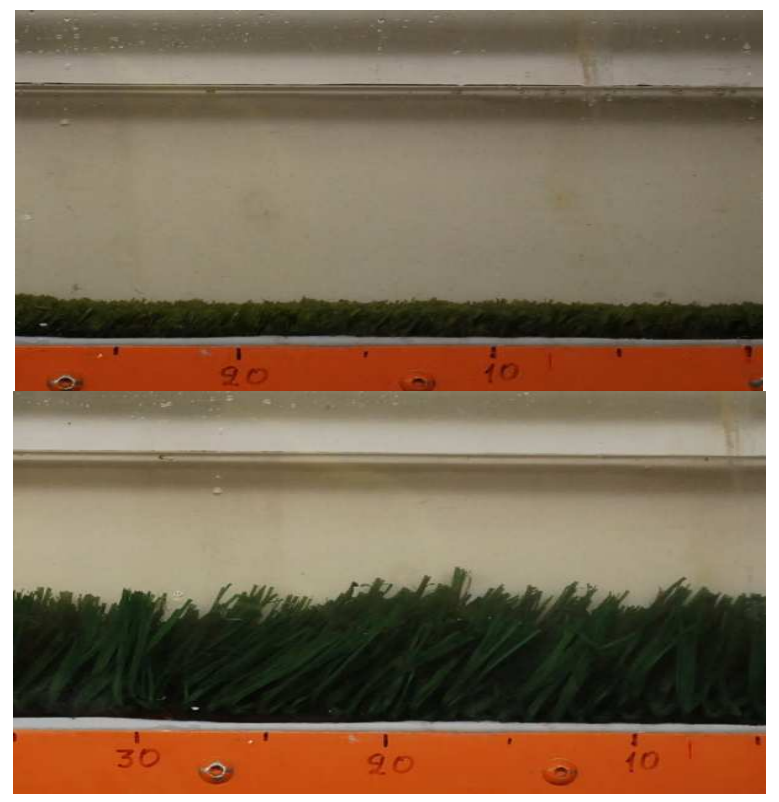

Photograph 1. Vegetation of : a) $2.5 \mathrm{~cm}$, and b) $5 \mathrm{~cm}$ height. 


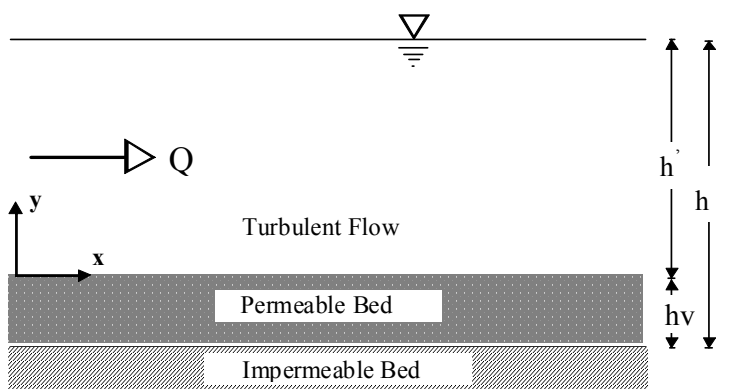

Figure 1. Geometrical characteristics of the flow over permeable bed.

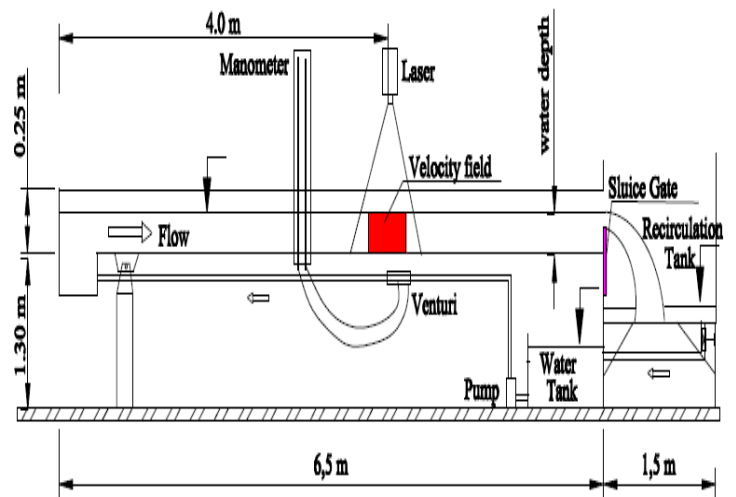

Figure 2. Experimental apparatus.

Table 1. Characteristics of the experiments.

\begin{tabular}{|c|c|c|c|c|}
\hline Experiment & $\begin{array}{l}\text { Total } \\
\text { flow } \\
\text { depth h (cm) }\end{array}$ & $\begin{array}{l}\text { Slope } \\
\text { S (\%o) }\end{array}$ & $\begin{array}{l}\text { Vegetation } \\
\text { Length } \\
\text { L (cm) }\end{array}$ & $\begin{array}{l}\text { Vegetation } \\
\text { Height } \\
h_{\mathrm{v}}(\mathrm{cm})\end{array}$ \\
\hline 1 & 10 & 2 & 150 & 2.5 \\
\hline 2 & 15 & 2 & 150 & 2.5 \\
\hline 3 & 10 & 4 & 150 & 2.5 \\
\hline 4 & 15 & 4 & 150 & 2.5 \\
\hline 5 & 10 & 6 & 150 & 2.5 \\
\hline 6 & 15 & 6 & 150 & 2.5 \\
\hline 7 & 10 & 2 & 300 & 2.5 \\
\hline 8 & 15 & 2 & 300 & 2.5 \\
\hline 9 & 10 & 4 & 300 & 2.5 \\
\hline 10 & 15 & 4 & 300 & 2.5 \\
\hline 11 & 10 & 6 & 300 & 2.5 \\
\hline 12 & 15 & 6 & 300 & 2.5 \\
\hline 13 & 10 & 2 & 150 & 5.0 \\
\hline 14 & 15 & 2 & 150 & 5.0 \\
\hline 15 & 10 & 4 & 150 & 5.0 \\
\hline 16 & 15 & 4 & 150 & 5.0 \\
\hline 17 & 10 & 6 & 150 & 5.0 \\
\hline 18 & 15 & 6 & 150 & 5.0 \\
\hline 19 & 10 & 2 & 300 & 5.0 \\
\hline 20 & 15 & 2 & 300 & 5.0 \\
\hline 21 & 10 & 4 & 300 & 5.0 \\
\hline 22 & 15 & 4 & 300 & 5.0 \\
\hline 23 & 10 & 6 & 300 & 5.0 \\
\hline 24 & 15 & 6 & 300 & 5.0 \\
\hline
\end{tabular}

Measurements of velocity were taken for inclined chan- nel slope. PIV is an optical method of fluid visualisation and is used to obtain instantaneous velocity measurements and related properties in fluids. The fluid is seeded with tracer particles which, for the purposes of PIV, are generally assumed to faithfully follow the flow dynamics. The motion of the seeding particles is used to calculate the velocity profile of the flow. Other techniques used to measure flows are Laser Doppler velocimetry and Hot-Film anemometry. The main difference between PIV and those techniques is that the former produces two dimensional vector fields, while the other techniques measure the velocity at a point. PIVs use the particle concentration method to identify individual particles in an image and follow their flow; however, tracking particles between images is not always a straightforward task. Individual particles could be "followed" when the particle concentration is low, a method called particle tracking velocimetry, whereas laser speckle velocimetry is used for cases where the particle concentration is high. The experimental uncertainty of the measured velocity with this technique is approximately \pm $2 \%$.

The measurements were conducted at a $4 \mathrm{~m}$ distance from the channel's entrance and above the top of the vegetation, where the flow is considered fully developed. The full development of the flow was evaluated comparing the velocity distributions above the vegetation in two vertical sections with a $60 \mathrm{~cm}$ separation distance. The uniformity of the flow was checked measuring the flow depth with point gauges at two cross-sections ( $4 \mathrm{~m}$ between the two sections). The desirable flow depth in the downstream section could be controlled using a weir at the channel's outlet. The error of the measured flow depth with the point gauge was $\pm 0.1 \mathrm{~mm}$.

\section{Analysis Of Results}

The current analysis aims to identify the impact of the channel slope, the vegetation height, the vegetation length and the relative vegetation depth on the characteristics of the flow. Figure 3 shows the variation of flow depth $h$ as regards the channel slope $S=4 \%$ and the vegetation length $\mathrm{L}=300 \mathrm{~cm}$. Before the vegetation $(\mathrm{x}=-10 \mathrm{~cm})$ the flow depth $h$ has the maximum value. At the point $x=-2 \mathrm{~cm}$ (before the vegetation) the flow depth reduced normally but at the point $\mathrm{x}=10 \mathrm{~cm}$ (inside the vegetation) the variation of flow depth is more abrupt (subcritical flow). The reduction continues normally inside the vegetation $(x=60,100,150$, $200,250 \mathrm{~cm})$. After the end of the vegetation the depth flow has a small abrupt reduction. At the end of vegetation $(x=300 \mathrm{~cm})$ the flow depth has a value of $2.0 \mathrm{~cm}$ lower than the initial measurement at the point $x=-10 \mathrm{~cm}(20 \%$ reduction). Figure 4 illustrates the impact of channel slope on the velocity distribution for $\mathrm{h}=10 \mathrm{~cm}$ and $\mathrm{hv}=5 \mathrm{~cm}$. Velocity increases when the channel slope increases from $2 \%$ to $6 \%$. 


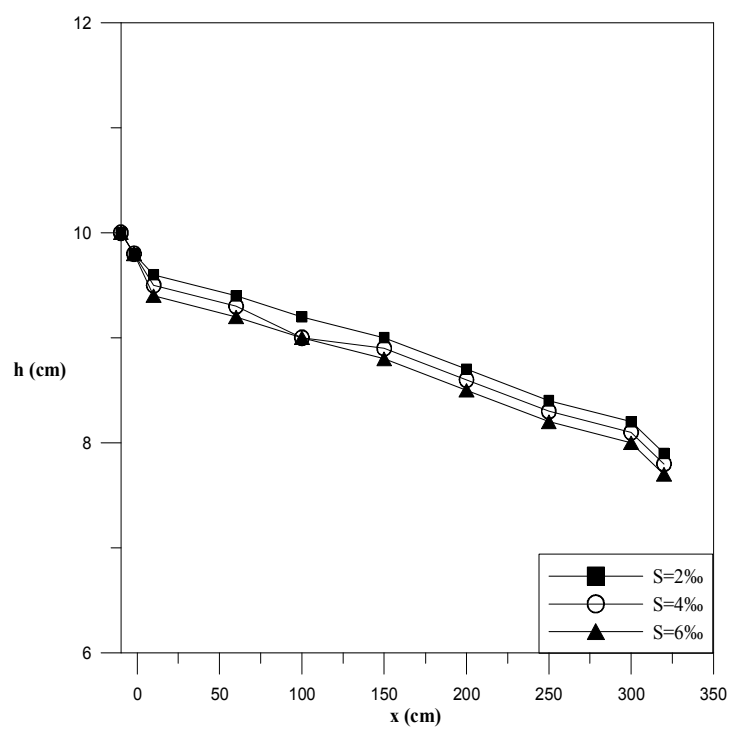

Figure 3. Impact of channel slope on depth flow for $h=10 \mathrm{~cm}$ and $\mathrm{hv}=5$ $\mathrm{cm}$.

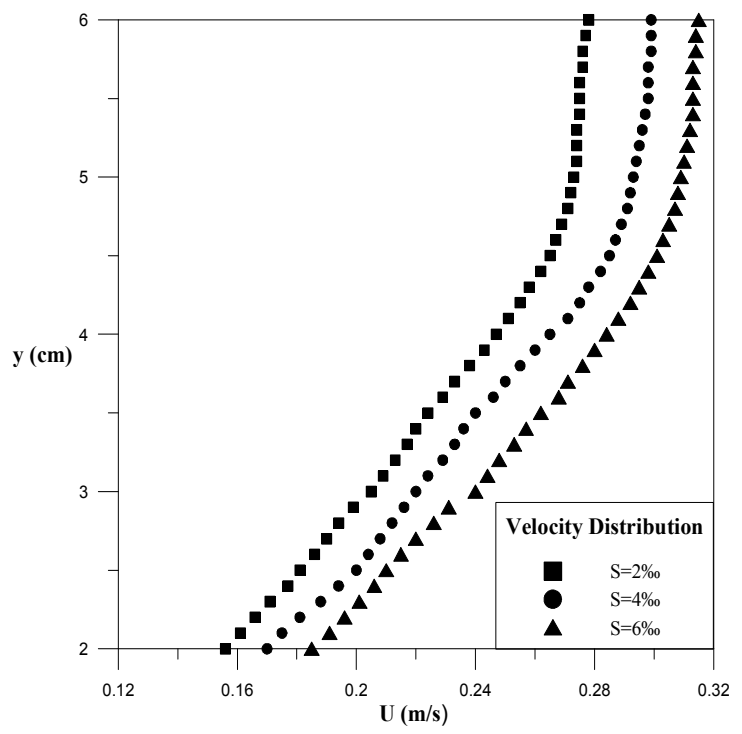

Figure 4. Impact of channel slope on velocity distribution for $h=10 \mathrm{~cm}$ and $h_{v}=5 \mathrm{~cm}$.

Figure 5 shows the impact of vegetation height hv to the velocity distribution for the same slope $(\mathrm{S}=2 \% \mathrm{o})$ and the same flow depth $(\mathrm{h}=10 \mathrm{~cm})$. The vegetation with the major height $(5 \mathrm{~cm})$ reduces significantly the velocities over the vegetation bed when compared with these with vegetation of $2.5 \mathrm{~cm}$. In addition, results show a region of zero velocity; between until $2.5 \mathrm{~cm}$ and $1 \mathrm{~cm}$ for a $5 \mathrm{~cm}$ and $2.5 \mathrm{~cm}$ vegetation respectively. This result shows that $50 \%$ of the vegetation behaves like an impermeable bed.

Figure 6 illustrates the impact of vegetation length on the flow depth. For the same flow depth $(\mathrm{h}=15 \mathrm{~cm})$, for the same slope $(\mathrm{S}=4 \% \mathrm{c})$ and for the same vegetation height $(\mathrm{hv}=5 \mathrm{~cm})$. Before the vegetation $(\mathrm{x}=-10 \mathrm{~cm})$ the flow depth $h$ for the vegetation length $L=150 \mathrm{~cm}$ is grater than the respectively with vegetation length $L=300 \mathrm{~cm}$. At the point $\mathrm{x}=10 \mathrm{~cm}$ (inside the vegetation) the variation of flow depth is more abrupt but also the flow depth $\mathrm{h}$ for the vege- tation length $\mathrm{L}=150 \mathrm{~cm}$ is grater than the respectively with vegetation length $L=300 \mathrm{~cm}$. The vegetation length reduces the flow depth. This is due to the additional resistance which induces the presence of the vegetation to the flow.

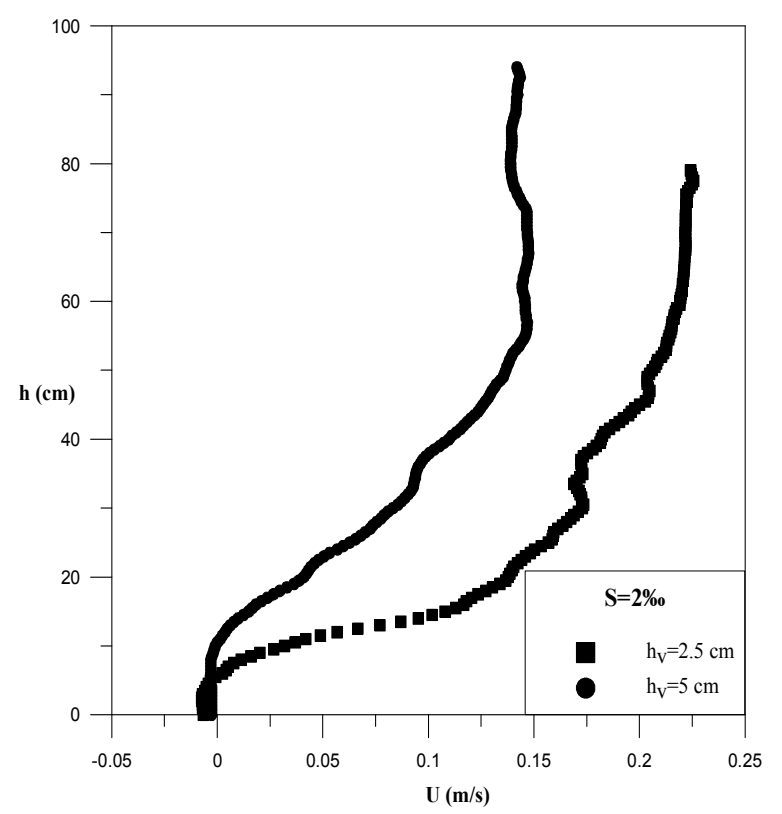

Figure 5. Impact of vegetation height on velocity distribution for $h=10$ $\mathrm{cm}$.

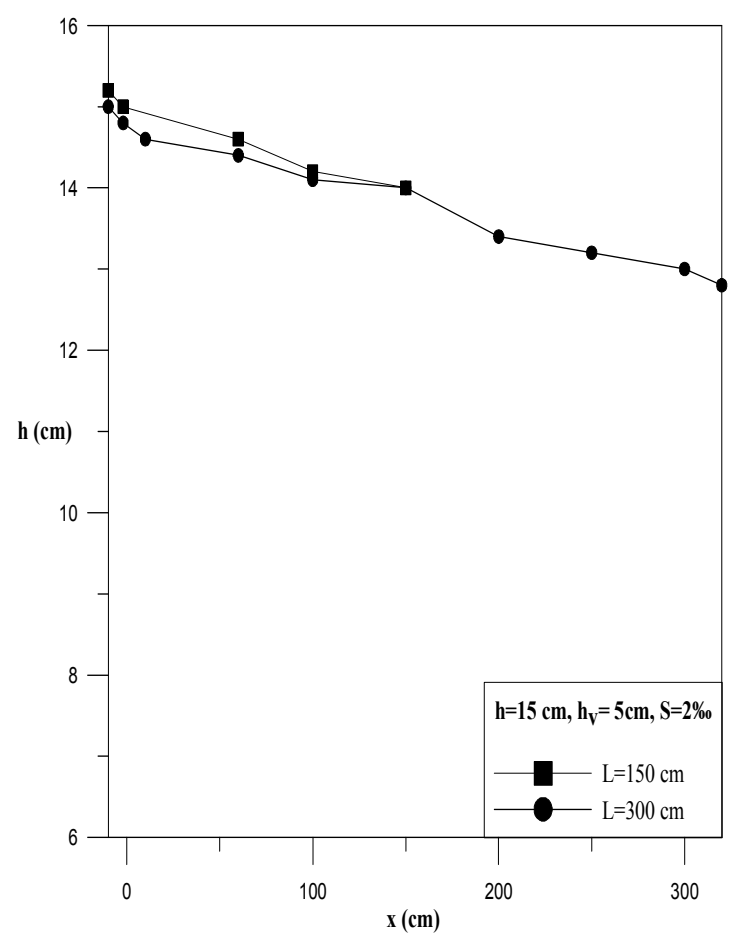

Figure 6. Impact of vegetation length on flow depth for $h=15 \mathrm{~cm}$., $h_{v}=5$ $\mathrm{cm}$ and $S=4 \%$.

Figure 7 shows the velocity distribution above the vegetation for $\mathrm{hv}=2.5 \mathrm{~cm}(\mathrm{~h} / \mathrm{hv}=6$ and 4$)$ and $\mathrm{hv}=5 \mathrm{~cm}(\mathrm{~h} / \mathrm{hv}=3$ and 2). The logarithmic law for flow over a solid, impermeable bed with depth $\mathrm{h}$ is also illustrated. We use dimensionless velocities using the friction velocity $\mathrm{U}^{*}$ 


$$
\left(U_{*}=\sqrt{\tau_{0} / \rho}\right)
$$

where $\tau 0$ is shear stress

$$
\left(\tau_{0}=\mu \frac{\partial u}{\partial y}\right)
$$

$\mu$ is dynamic viscosity and $\rho$ is the water density) and the distance from the interface $y$ with the ratio $U^{*} / v$. This normalization allows us to evaluate the applicability of the logarithmic law for these conditions. Velocities are below the logarithmic law line which indicates that velocities above vegetation are reduced in comparison with velocities over an impermeable bed. In particular, the velocities are reduced with decreasing $\mathrm{h} / \mathrm{hv}$ ratio due to the greater influence of the vegetation on the flow above it.

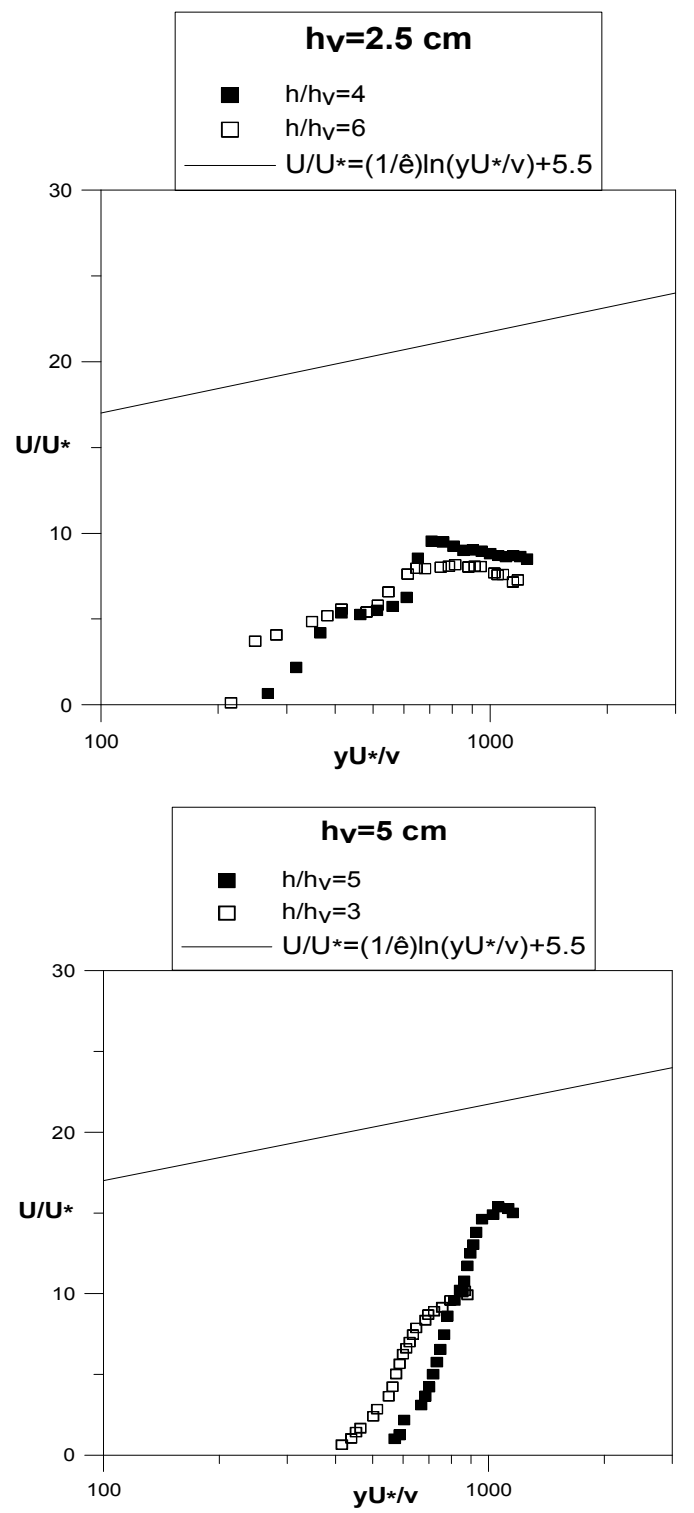

Figure 7. Dimensionless velocity distribution for $S=2 \%, L=150 \mathrm{~cm}$ and vegetation height (hv) equal to: a) $2.5 \mathrm{~cm}$, and b) $5 \mathrm{~cm}$.

\section{Conclusions}

In this study, the impact of vegetation on the characteristics of the flow in an inclined open channel is studied experimentally using a Particle Image Velocimetry (PIV). For the simulation of porous bed, flexible vegetation with grass with different height $(2.5 \mathrm{~cm}$ and $5 \mathrm{~cm})$ with the same porosity ( $\varepsilon=0.75$ ) were used to represent the vegetated bed. The following conclusions can be derived:

The impact of channel slope is very significant on the flow depth and the velocity profile. Before the vegetation the flow depth $h$ has the maximum value. Just before the vegetation the flow depth reduced normally but inside the vegetation the variation of flow depth is more abrupt (subcritical flow). After the end of the vegetation the depth flow has a small abrupt reduction. At the end of vegetation the flow depth has a value of $2.0 \mathrm{~cm}$ lower than the initial measurement before the vegetation ( $20 \%$ reduction). Also velocity increases when the channel slope increases from $2 \%$ to $6 \%$.

The impact of vegetation height is very significant on the velocity profile. The vegetation with the major height $(5 \mathrm{~cm})$ reduces significantly the velocities over the vegetation bed when compared with these with vegetation of 2.5 $\mathrm{cm}$. In addition, results show a region of zero velocity; between until $2.5 \mathrm{~cm}$ and $1 \mathrm{~cm}$ for a $5 \mathrm{~cm}$ and $2.5 \mathrm{~cm}$ vegetation respectively. This result shows that $50 \%$ of the vegetation behaves like an impermeable bed.

The impact of vegetation length on the flow depth is significant. The vegetation length reduces the flow depth. This is due to the additional resistance which induces the presence of the vegetation to the flow.

The velocities are reduced with decreasing $\mathrm{h} / \mathrm{hv}$ ratio due to the greater influence of the vegetation on the flow above it.

\section{References}

[1] Beavers, G. S., Joseph, D. D., Boundary Conditions at a Naturally Permeable Wall, J. Fluid Mech., 30(1), 197-207, 1967.

[2] Bigillon, F., Nino, Y., and Garcia, M.H., Measurements of turbulence characteristics in an open-channel flow over a transitionally-rough bed using particle image velocimetry. Experiments in Fluids, 41 (6), 857-867, 2006.

[3] Carollo, F.G., Ferro, V. and Termini, D., Flow resistance law in channels with flexible submerged vegetation, J. of Hydraulic Engineering, 131(7), pp. 554-564, 2005.

[4] Chan, H.C., Leu, J.M., Lai, C.J. and Yafei Jia, Turbulent flow over a channel with fluid-saturated porous bed, Journal of Hydraulic Engineering, ASCE, vol. 133, no. 6, pp 610$617,2007$.

[5] Jarvela, J., Effect of submerged flexible vegetation on flow structure and resistance, J. of Hydrology, 307, pp. 233-241, 2005.

[6] Pechlivanidis G., Keramaris E., Pechlivanidis I., Measure- 
ments of Turbulent Characteristics in an Open Channel Using PIV (Particle Image Velocimetry), Global Nest Journal, Volume 14 (3), pp. 378-385, 2012.

[7] Poulikakos, D., Kazmierczak, M., Forced Convection in a Duct Partially Filled with a Porous Material, J. of Heat Transfer, 109, pp. 653-662, 1987.

[8] Prinos, P., Sofialidis D., Keramaris E., Turbulent flow over and within a porous bed, Journal of Hydraulic Engineering, ASCE, vol. 129, pp. 720-733, 2003.

[9] Stephan, U., Gutknecht, D., Hydraulic resistance of sub- merged flexible vegetation, Journal of Hydrology, vol. 269, (1/2), pp. 27-43, 2002.

[10] Roussinova, V., Shinneeb, A-M. and Balachandar, R., Investigations of fluid structures in a smooth open-channel flow using proper orthogonal decomposition, J. of Hydraulic Engineering, 136(3), pp. 143-154, 2010.

[11] Wilson, C.A.M.E, Stoesser, T., Bates, P.D. and Batemann Pinzen, A., Open channel flow through different forms of submerged flexible vegetation, J. of Hydraulic Engineering, 129(11), pp. 847-853, 2003. 